\title{
EFEITO DA ESPESSURA DO ADESIVO NO COMPORTAMENTO MECÂNICO DE JUNTAS ADESIVAS PARA APLICAÇÃO AUTOMOTIVA
}

\author{
Diego Tolotti de Almeida ' \\ João Henrique Corrêa de Souza ' \\ Piero David Maehler ' \\ Tiago Roberto Simon '
}

\begin{abstract}
Resumo
É crescente a aplicação de adesivos estruturais como método de fixação nos diversos segmentos da indústria, principalmente na indústria automotiva. Isso se justifica pela necessidade de redução do consumo de energia e economia de recursos, trazendo assim, uma série de benefícios ao produto/processo, quando comparado aos tradicionais sistemas de fixação com parafuso, rebite ou solda. Esta pesquisa estudou alguns fatores que influenciam nas forças mecânicas das juntas adesivas submetidas ao ensaio de tração cisalhante (Lap Shear) e tração cruzada (Cross Peel). Três diferentes espessuras do adesivo foram utilizadas para fabricar as juntas adesivas $(0,80, I, 50$ e 3,00 mm). Além disso, foi possível identificar o efeito de diferentes materiais de base sobre o comportamento mecânico das juntas adesivas. Resultados experimentais mostraram que menores espessuras de adesivo aumentam consideravelmente a resistência mecânica das juntas submetidas a ensaio de tração cisalhante. Por outro lado, resultados obtidos no ensaio de tração cruzada, não apresentaram este mesmo comportamento. No entanto, existe uma relação entre a performance da junta e o material do substrato a ser colado. Palavras-chave: Juntas adesivas; Comportamento mecânico; Resistência ao cisalhamento; Tração cruzada; Espessura do adesivo.
\end{abstract}

\section{EFFECT OF ADHESIVE THICKNESS IN THE MECHANICAL BEHAVIOR OF ADHESIVE JOINTS FOR AUTOMOTIVE APPLICATIONS}

\begin{abstract}
The application of structural adhesives is increasing as a method of fixation in the various segments of industry, especially in the automotive industry. This is justified by the need to reduce energy consumption and save resources, thus bringing a series of benefits to the product / process compared to traditional fastening systems with bolt, rivet or weld. This study studied some factors that influence the mechanical strength of the adhesive shear tests submitted to the Lap Shear and Cross Peel tests. Three different adhesive thicknesses were used to make the adhesive joints $(0.80,1.50$ and $3.00 \mathrm{~mm})$. In addition, it was possible to identify the effect of different base materials on the mechanical behavior of the adhesive joints. Experimental results have shown that minor adhesive thicknesses considerably increase the mechanical strength of the joints subjected to shear traction tests. On the other hand, the results of the cross-traction test did not obtain this same behavior. However, there is a relationship between the performance of the joint and the material of the substrate to be bonded.
\end{abstract}

Keywords: Adhesive seals; Mechanical behavior; Shear strength; Cross traction; Adhesive thickness.

\section{INTRODUÇÃO}

A necessidade de melhorar os métodos de uniões em aplicações estruturais dos veículos automotores, tem possibilitado a pesquisa em processos diferentes dos sistemas de uniões tradicionais, como a soldagem ou rebitagem [I].
A união através da aplicação de adesivos estruturais tem aumentado, pois oferece vantagens como a redução de massa, redução/eliminação de concentradores de tensões, comportamento ao impacto, absorção de energia, menor

'Bruning Tecnometal, Panambi, RS, Brasil.E-mail: diegot@bruning.com.br 
vibração e amortecimento do som [2]. Contudo, a obtenção destas vantagens requer um adesivo específico designado para o projeto, melhorando a performance do produto e restringindo algumas limitações [3]. A análise das principais contribuiç̧̃os sobre a concepção das juntas adesivas, juntamente com os resultados dos estudos, permite o planejamento estruturado do projeto e a seleção do melhor adesivo $[4,5]$.

Para garantir a segurança do usuário final, os engenheiros de produto devem ter informações suficientes sobre as propriedades mecânicas do material, antes de introduzir o material na especificação do produto [6]. Além das propriedades do material de base, a performance mecânica das juntas adesivas com estes materiais, devem ser suficientemente caracterizadas. As propriedades mecânicas das juntas adesivas têm sido estudadas, e muitos pesquisadores definem usando os ensaios de tração cisalhante, duplo tração cisalhante, tração cruzada, junta de topo e teste do componente [6-10].

As pesquisas têm caracterizado as propriedades mecânicas das juntas adesivas por vários métodos de teste, $\mathrm{e}$ mostram que a resistência da junta depende da configuração, modo de carga, taxa de carregamento, condições ambientais e espessura do adesivo [II,I2].

Schijve et al. [13] constataram que o aumento da espessura do adesivo em chapas de alumínio da liga 2024-T3 proporcionavam um melhor comportamento à fadiga. Segundo Her [14], quanto mais fina for a camada adesiva, maior será a tensão cisalhante. Li et al. [15] mostraram a distribuição da tensão de descascamento para diferentes espessuras de adesivo, onde menores espessuras adesivas conduziram a maiores tensões. Pandey [16] estudou sobre análise não linear por elementos finitos de juntas de cisalhamento, considerando a viscoplasticidade em adesivos, constatando que os picos de tensão verificados decrescem com o aumento da espessura da camada adesiva. Bezemer apud Vaidya [17] cita que menores espessuras do adesivo proporcionam uma ótima colagem para testes estáticos ou de média velocidade.

Muitos autores reportam valores de espessuras típicas para as regiões de colagem, embora se saiba que esta escolha está intimamente ligada às propriedades do adesivo, como por exemplo, viscosidade, molhabilidade e composição química. Além da espessura do adesivo, uma característica muito importante que deve ser levada em consideração é a ausência de descontinuidades como bolhas e vazios no interior da junta adesiva.

Neste estudo, primeiro foram selecionados os materiais que são comumente usados em veículos automotores, isto é, liga de alumínio 5052H32, chapas de aço NBR 5915 e GMW3032. Em seguida, as juntas adesivas foram fabricadas unindo materiais similares, ou seja, aço com aço e alumínio com alumínio, com três diferentes espessuras da camada do adesivo $(0,80 \mathrm{~mm}, 1,50 \mathrm{~mm}$ e $3,00 \mathrm{~mm})$. Os desempenhos mecânicos estáticos das juntas foram avaliados pelos ensaios de tração por cisalhamento (Lap Shear) e tração cruzada (Cross Peel). As fraturas dos adesivos nas juntas foram analisadas afim de se obter a área exata da falha (coesiva ou adesiva). Com os resultados deste estudo, a equipe de pesquisa e desenvolvimento da empresa Bruning Tecnometal, pôde conhecer o potencial da adesão estrutural na união de chapas sobrepostas de mesmo material, e assim apresentar maneiras de otimizar os projetos com adesivo estrutural.

\section{MATERIAIS E MÉTODOS}

Neste estudo, foram utilizadas chapas de alumínio $5052 \mathrm{H} 32 \mathrm{com}$ I,95mm de espessura, chapas de aço NBR 5915 com I,50mm de espessura e chapas de aço GMW3032M com $1,00 \mathrm{~mm}$ de espessura. As dimensões geométricas dos corpos de prova para ensaio de Lap Shear seguiram o especificado pela norma ASTM D I002:94 [18] e Cross Peel seguiu o padrão da norma J I553:86 [19]. A Figura I apresenta detalhes dos corpos de prova, bem como a real área de adesão, que em todos os casos foi de $645,16 \mathrm{~mm}^{2}\left(\mathrm{I}\right.$ pol. $\left.{ }^{2}\right)$.

A composição química e propriedades mecânicas dos materiais utilizados podem ser visualizados na Tabela I.

Para a união das juntas, foi utilizado o adesivo acrílico bi-componente LORD 406/19. A Tabela 2 apresenta a composição química do Adesivo.
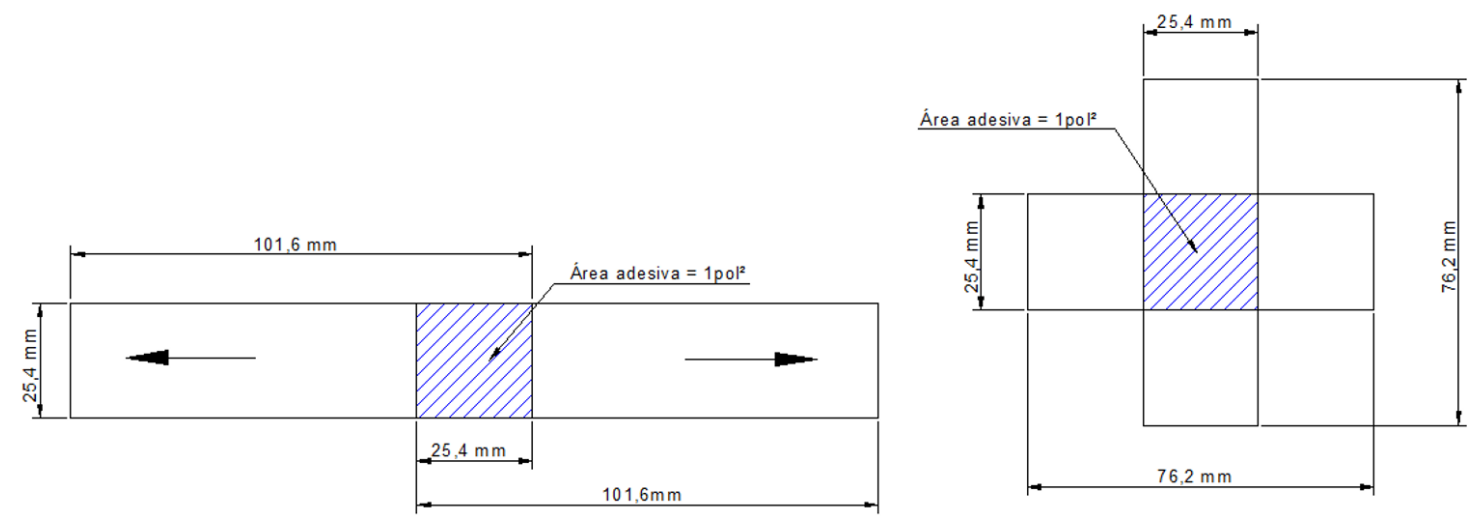

Figura I. Corpo de prova ensaio Lap Shear (esquerda), Cross Peel (direita). 
Tabela I. Composição química e propriedades mecânicas [20-23]

\begin{tabular}{|c|c|c|}
\hline \multicolumn{3}{|c|}{ AÇO NBR 59 I 5} \\
\hline Composição Química & Carbono - C (\%) & 0.13 MÁX. \\
\hline \multirow[t]{4}{*}{ (ABNT NBR 59I5) } & Manganes - Mn (\%) & 0.60 MÁX. \\
\hline & Fósforo - P (\%) & 0.040 MÁX. \\
\hline & Alumínio - Al (\%) & 0.010 MíN. \\
\hline & Enxofre - S (\%) & 0.040 MÁX. \\
\hline Propriedades Mecânicas & Limite Escoamento $\left(\mathrm{N} / \mathrm{mm}^{2}\right)$ & 280 MÁX. \\
\hline \multirow[t]{2}{*}{ (ABNT NBR 5915) } & Limite Resist. Tração $\left(\mathrm{N} / \mathrm{mm}^{2}\right)$ & $270-390$ \\
\hline & Along. (\%) Lo $=5,65 \times \sqrt{\mathrm{S}_{\mathrm{o}}}$ & 30 MíN. \\
\hline \multicolumn{3}{|c|}{ AÇO GMW3032 } \\
\hline Composição Química & Carbono - C (\%) & 0.12 MÁX. \\
\hline \multirow[t]{5}{*}{ (GMW 3032) } & Manganes - Mn (\%) & I.50 MÁX. \\
\hline & Silício - Si (\%) & 0.50 MÁX. \\
\hline & Fósforo - P (\%) & 0.030 MÁX. \\
\hline & Alumínio - Al (\%) & 0.015 MíN. \\
\hline & Enxofre - S (\%) & 0.030 MÁX. \\
\hline Propriedades Mecânicas & Limite Escoamento $\left(\mathrm{N} / \mathrm{mm}^{2}\right)$ & $340-420$ \\
\hline \multirow[t]{2}{*}{ (GMW 3032) } & Limite Resist. Tração $\left(\mathrm{N} / \mathrm{mm}^{2}\right)$ & 4I0 MíN. \\
\hline & Along. (\%) Lo $=5,65 \times \sqrt{S_{0}}$ & 22 MíN. \\
\hline \multicolumn{3}{|c|}{ ALUMÍNIO 5052 H32 } \\
\hline Composição Química & Manganes - Mn (\%) & 0.10 MÁX. \\
\hline \multirow[t]{4}{*}{ (ABNT NBR 6834) } & Silício - Si (\%) & 0.25 MÁX. \\
\hline & Alumínio - Al (\%) & RESTANTE \\
\hline & Cobre - Cu (\%) & 0.10 MÁX. \\
\hline & Cromo - Cr (\%) & $0.15-0.35$ \\
\hline Propriedades Mecânicas & Limite Escoamento $\left(\mathrm{N} / \mathrm{mm}^{2}\right)$ & I 30 MíN. \\
\hline \multirow[t]{2}{*}{ (ABNT NBR 7823) } & Limite Resist. Tração $\left(\mathrm{N} / \mathrm{mm}^{2}\right)$ & $210-260$ \\
\hline & Along. (\%) Lo $=5,65 \times \sqrt{S_{0}}$ & 7 MíN. \\
\hline
\end{tabular}

Tabela 2. Composição Química do Adesivo [24]

\begin{tabular}{|c|c|c|c|}
\hline \multicolumn{4}{|c|}{ Adesivo 406} \\
\hline Composição Química & Metacrilato de Metila & $\%$ em peso Menor que & 35,0 \\
\hline \multirow[t]{4}{*}{ (FISPQ LORD) } & Ácido Acrílico & & 10,0 \\
\hline & Ciclohexil Metacrilato & & 4,0 \\
\hline & Fosfato Ester Metacrilato & & 3,0 \\
\hline & N.N.-Dimetilanilina & & 2,0 \\
\hline \multicolumn{4}{|c|}{ Acelerador 19} \\
\hline Composição Química & Resina Epoxi & $\%$ em peso Menor que & 55,0 \\
\hline \multirow[t]{2}{*}{ (FISPQ LORD) } & Diisobutil Ftalato & & 4,0 \\
\hline & Peroxido de Benzoila & & 4,0 \\
\hline
\end{tabular}

A Tabela 3 apresenta algumas propriedades de cura do adesivo.

Para a realização do experimento, primeiramente foi realizado a preparação da superfície dos corpos de prova. A região da superfície que recebeu o adesivo foi limpa com acetona, com objetivo de remover graxas, contaminantes soltos ou óxidos. Após a preparação da superfície dos corpos de prova, o adesivo LORD 406/ 9 foi aplicado no substrato, com auxílio de uma pistola de aplicação pneumática, onde foi realizada a mistura e distribuição homogênea do adesivo.
De acordo com as recomendações do fornecedor, o tempo de movimentação dos corpos de prova, para ajustes e regulagem da espessura, é de até 6 minutos. Porém a cura completa é de 24 horas à temperatura ambiente. Desta forma, para garantir o alinhamento dos corpos de prova e a espessura desejada do filme adesivo, foi necessário projetar e fabricar um dispositivo de controle, que pode ser visualizado na Figura 2.

O dispositivo de colagem utilizado e confeccionado especialmente para o procedimento, recebeu uma fita 
Tabela 3. Propriedades de cura do adesivo [24]

\begin{tabular}{cccc}
\hline $\begin{array}{c}\text { Resistência à ruptura por } \\
\text { tração, ASTM D638 }\end{array}$ & $\begin{array}{c}\text { Alongamento, } \\
\text { ASTM D638 }\end{array}$ & $\begin{array}{c}\text { Módulo de Elasticidade, } \\
\text { ASTM D638 }\end{array}$ & $\begin{array}{c}\text { Temperatura de transição } \\
\text { vitrea, ASTM EI640-99 }\end{array}$ \\
\hline$[\mathrm{MPa}]$ & {$[\%]$} & {$[\mathrm{MPa}]$} & {$\left[{ }^{\circ} \mathrm{C}\right]$} \\
32,1 & 30 & 896,3 & 72 \\
\hline
\end{tabular}

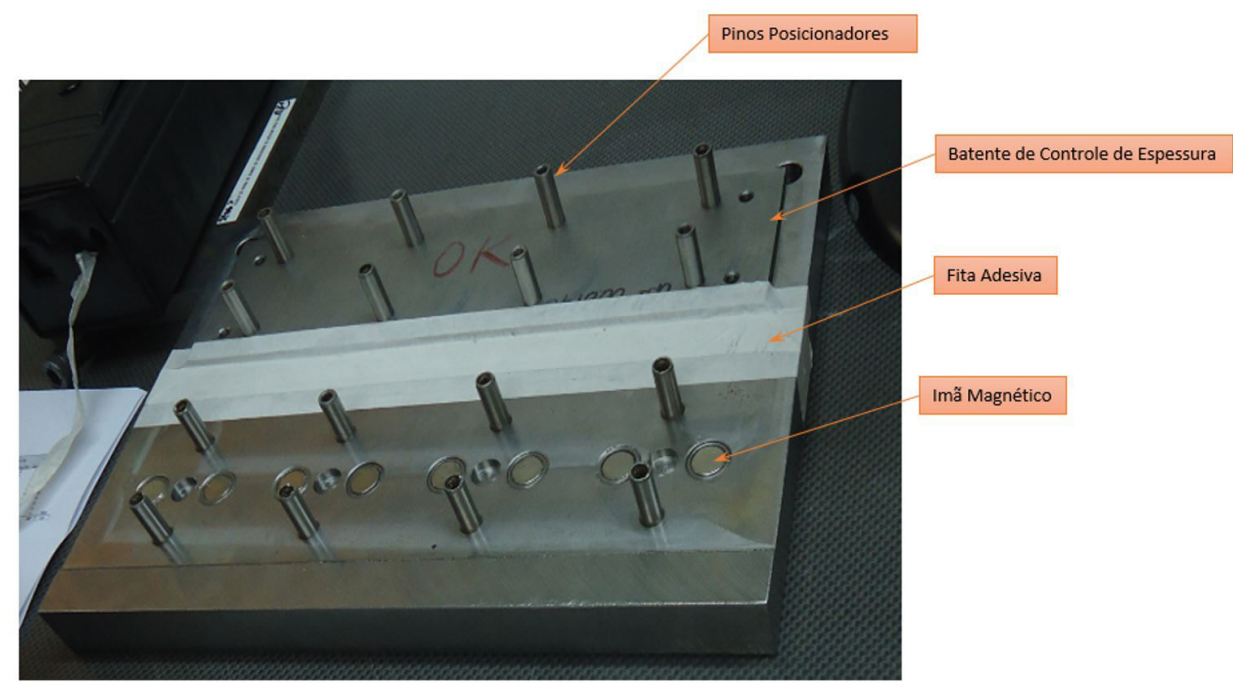

Figura 2. Dispositivo de colagem.

adesiva no centro da região de colagem, de forma a facilitar a posterior retirada de excesso de adesivo que viesse a aderir na superfície do dispositivo. A colagem foi efetuada de forma a molhar a superfície do corpo de prova, primeiramente posicionando o corpo de prova da parte inferior e fazendo o encaixe da junta cuidadosamente com a parte superior. Uma espátula foi utilizada para retirada do excesso do adesivo. Em seguida, contrapesos foram adicionados em suas posições no dispositivo, de forma a pressionar a camada de cola, conduzindo-se à espessura prevista.

Com a finalidade de avaliar o efeito das propriedades mecânicas das juntas coladas, foi priorizada a aplicação de diferentes espessuras do adesivo, de modo a definir a ótima espessura para cada condição dos materiais testados. A Tabela 4 resume os parâmetros empregados no processo de colagem.

Cada parâmetro produziu cinco corpos de prova segundo os parâmetros definidos na Tabela 4, totalizando 90 amostras. Todo procedimento de colagem foi realizado em ambiente com temperatura e umidade relativa do ar controladas $\left(25^{\circ} \mathrm{C}\right.$ e UR $\left.50 \%\right)$.

Os ensaios mecânicos de tração cisalhante (Lap Shear) e tração cruzada (Cross Peel) foram realizados em uma máquina de ensaio universal da marca EMIC, modelo DL 20000, com capacidade de carga de 20KN.

Após a realização dos ensaios destrutivos, foi caracterizado as falhas encontradas em cada combinação de parâmetros, onde foram caracterizados os modos de falhas. A falha coesiva ocorre quando há rompimento das ligações
Tabela 4. Parâmetros no processo de colagem

\begin{tabular}{|c|c|c|c|}
\hline Ensaio & Material & $\begin{array}{c}\text { Espessura } \\
\text { Adesivo }\end{array}$ & Repetições \\
\hline [Tipo] & [Esp.] & {$[\mathrm{mm}]$} & [\#] \\
\hline \multirow[t]{9}{*}{ Lap Shear } & $5052 \mathrm{H} 32$ & 0,80 & 5 \\
\hline & NBR 59I5 & & 5 \\
\hline & GMW3032 & & 5 \\
\hline & $5052 \mathrm{H} 32$ & $\mathrm{I}, 50$ & 5 \\
\hline & NBR 59I5 & & 5 \\
\hline & GMW3032 & & 5 \\
\hline & $5052 \mathrm{H} 32$ & 3,00 & 5 \\
\hline & NBR 59I5 & & 5 \\
\hline & GMW3032 & & 5 \\
\hline \multirow[t]{9}{*}{ Cross Peel } & $5052 \mathrm{H} 32$ & 0,80 & 5 \\
\hline & NBR 59I5 & & 5 \\
\hline & GMW3032 & & 5 \\
\hline & $5052 \mathrm{H} 32$ & 1,50 & 5 \\
\hline & NBR 59I5 & & 5 \\
\hline & GMW3032 & & 5 \\
\hline & $5052 \mathrm{H} 32$ & 3,00 & 5 \\
\hline & NBR 5915 & & 5 \\
\hline & GMW3032 & & 5 \\
\hline
\end{tabular}

do adesivo, ou seja, na qual a trinca se inicia e se propaga na própria camada adesiva. A falha adesiva se dá quando ocorre o rompimento das ligações de Van der Waals, em que acontece a fratura entre o adesivo e o substrato, na qual 
a trica se inicia e se propaga na interface. E a falha mista, que é uma mistura parcialmente da falha coesiva e adesiva.

\section{RESULTADOS E DISCUSSÃO}

A partir de ensaios de comportamento estrutural das uniões coladas, foram processados os resultados levando-se em consideração aspectos como o perfil do gráfico tensão-deformação obtido nos ensaios mecânicos de cisalhamento, onde foram excluídos durante o processamento dos dados os resultados que apresentaram regiões não coladas, defeitos pontuais ou mesmo escorregamento das garras durante o ensaio mecânico, pois estes eventos conduzem a valores de resultados que não representam o estado real de resistência da junta colada sob estudo.

A Figura 3 apresenta a média e o desvio padrão referente às medidas de cada um dos grupos analisados com diferentes espessuras do adesivo, referentes à tensão obtida no ensaio de tração cisalhante (lap shear).

É importante ressaltar que cada uma das juntas confeccionadas teve a mesma área de colagem, correspondente a $645,16 \mathrm{~mm}^{2}$ (I pol. ${ }^{2}$ ), a partir deste valor, foram calculados os resultados de tensão de ruptura das juntas.

A Figura 3 mostra que existe uma relação linear descendente dos resultados com o aumento da espessura do adesivo, ou seja, quanto maior é a espessura do adesivo, menor é a tensão de ruptura das juntas, mesmo para variações entre os diferentes materiais. Mahdi et al. [25] e Naito et al. [26] verificaram experimentalmente o mesmo comportamento.

Adams e Peppiatt [27] propuseram três justificativas possíveis para explicar a diminuição da resistência da junta com o aumento da espessura do adesivo: maiores concentrações de tensões, maior probabilidade da presença de defeitos internos (porosidade, vazios e microfissuras devido às tensões residuais induzidas pelo processo de cura), e taxa de deformação inferior. Os autores concluíram ainda que a presença dos defeitos internos é o fator preponderante na explicação do fenômeno. Para Gleich et al. [28] a diminuição da resistência da junta é baseada nas tensões interfaciais. $\mathrm{O}$ autor demostrou que as juntas com menor valor de espessura do adesivo, apresentam uma distribuição mais uniforme para ambas as componentes de tensão.

É possível observar, que o aumento da espessura do adesivo conduziu a uma diminuição da tensão de ruptura dos materiais, sendo esta redução mais significativa para o material GMW 3032. Nota-se um elevado desvio padrão nestas amostras, pois o aço galvanizado como é o caso do GMW 3032, possui uma camada de tratamento químico (deposição de zinco) que diminui a tensão superficial e cria um elemento intermediário ao material de base, comprometendo a adesão. Segundo Fruet [29] pode-se melhorar a tensão superficial do aço galvanizado com a utilização de primers para neutralizar a superfície, mas o processo produtivo da chapa de aço galvanizado é o ponto mais importante que deve ser observado e controlado.

Também é possível observar, que os valores de tensão obtidos com o alumínio são os maiores em todas as condições analisadas, sendo em média I $2 \%$ maior quando comparado com o aço NBR59I5 e 23\% maior quando comparado com o aço galvanizado GMW 3032. Este fato, pode ser explicado devido a existência de irregularidades e sujidades na superfície dos aços NBR 5915 e GMW 3032, que podem constituir pontos de iniciação de trincas.

Bikerman [30] sugere que a inclusão de gases, formação de filme de óxidos e concentração de constituintes em pequenas proporções, favorecem a formação da camada fraca. Ele sugere que além de uma boa preparação superficial dos corpos de prova a serem colados, um ponto importante é diminuir a rugosidade superficial. As irregularidades superficiais causam bolsões de ar, que favorecem a diminuição da resistência da junta e assim se comportam como pontos iniciadores de trincas. Isto explica, o fato de somente as amostras de alumínio serem as únicas que apresentaram falha totalmente coesiva, devido ao alumínio possuir uma rugosidade superficial menor, quando comparado ao aço.

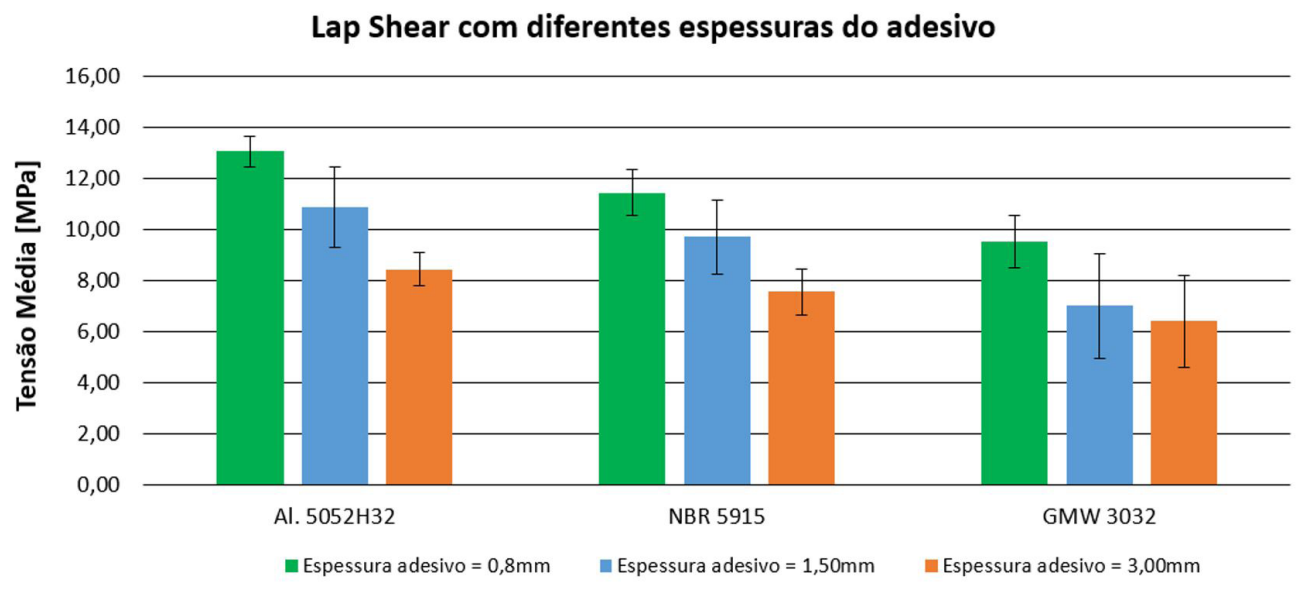

Figura 3. Ensaio de tração cisalhante (lap shear). 
$\mathrm{Na}$ Figura 4, pode-se verificar um exemplo dos modos de falhas ocorridos nos corpos de prova submetidos ao ensaio de tração cisalhante (lap shear).

A Figura 5 apresenta o gráfico onde é possível visualizar os diferentes modos de falhas ocorridos nos corpos de prova. As falhas são caracterizadas como coesiva e adesiva e estão apresentadas na forma de porcentagem de área em que cada falha ocorreu, correspondente a uma área colada de $645,16 \mathrm{~mm}^{2}$ (I pol. $\left.{ }^{2}\right)$.

O substrato em alumínio apresentou em todas as espessuras do adesivo falha $100 \%$ coesiva. Isso indica que a ligação entre o adesivo e o substrato é mais forte que do que a própria resistência interna do adesivo. Este modo de falha nos indica também que a preparação da superfície dos substratos está correta. No entanto, as amostras de aço, possuem regiões com falha mista, ou seja, coesiva e adesiva. É perceptível nesta condição que quanto maior é a espessura do adesivo, maior é a porcentagem de falha adesiva e menor a de falha coesiva. Sendo que para condição com espessura do adesivo de $0,80 \mathrm{~mm}$ a falha adesiva representa $55 \%$ da área do corpo de prova, quando comparado com a espessura do adesivo de $3,00 \mathrm{~mm}$, que a falha adesiva representa $96 \%$ da área colada do corpo de prova.

A Figura 5 demostra que na condição do substrato zincado GMW 3032 com as três diferentes espessuras o modo de falha foi totalmente adesiva, ou seja, quando o adesivo se solta do substrato, deixando a superfície limpa. Este modo de falha é indesejável à união, pois a trinca se inicia e se propaga na interface do adesivo e substrato, reduzindo assim as tensões de cisalhamento da junta. Segundo Taib et al. [3I], a espessura da camada adesiva é um fator importante, sendo que uma fina camada adesiva estará mais suscetível a um estado plano de tensões do que a um estado plano de deformações. $O$ aumento da camada adesiva aumenta a

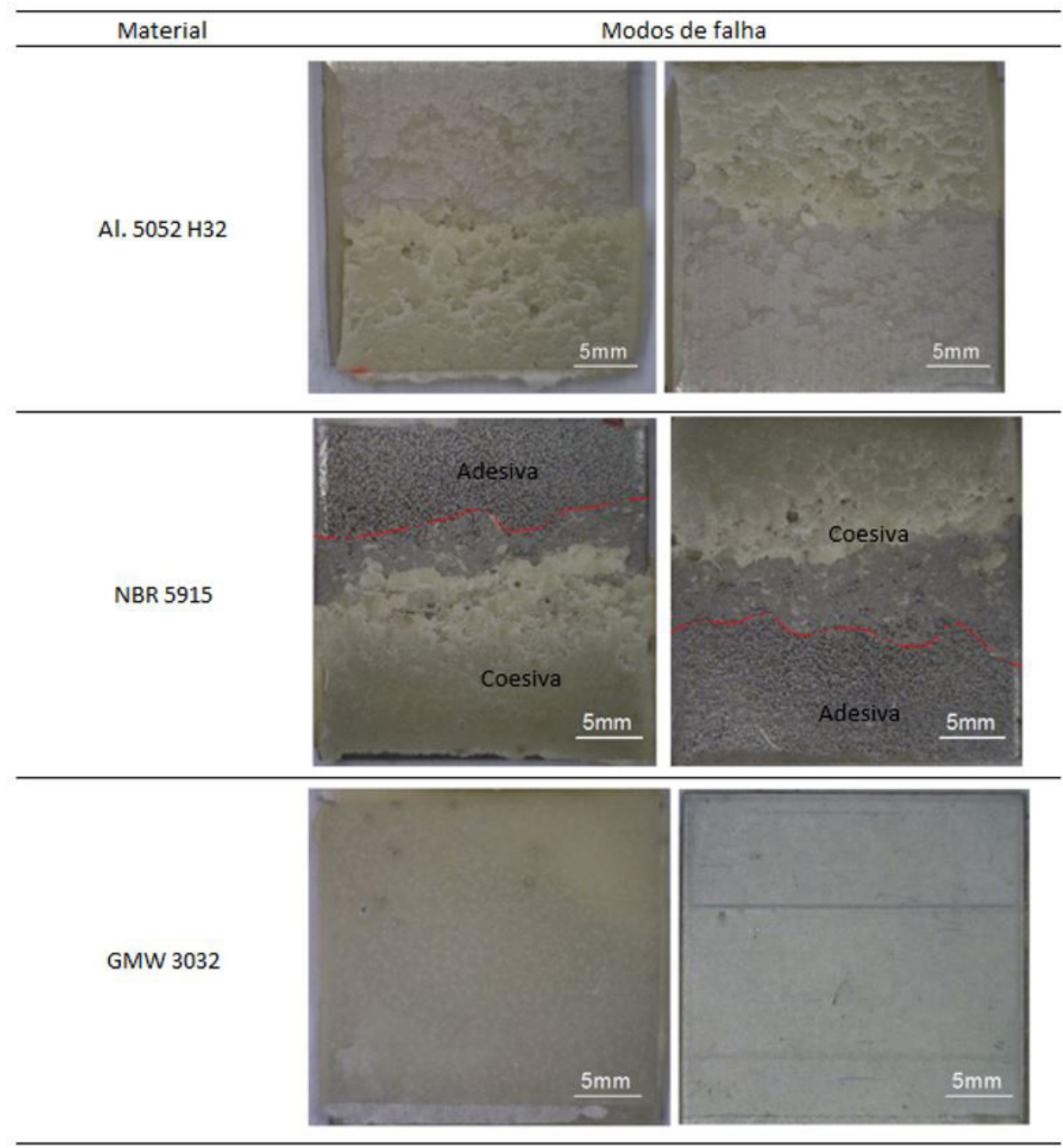

Figura 4. Modos de falhas de tração cisalhante (lap shear). 
Lap Shear Modos de Falhas

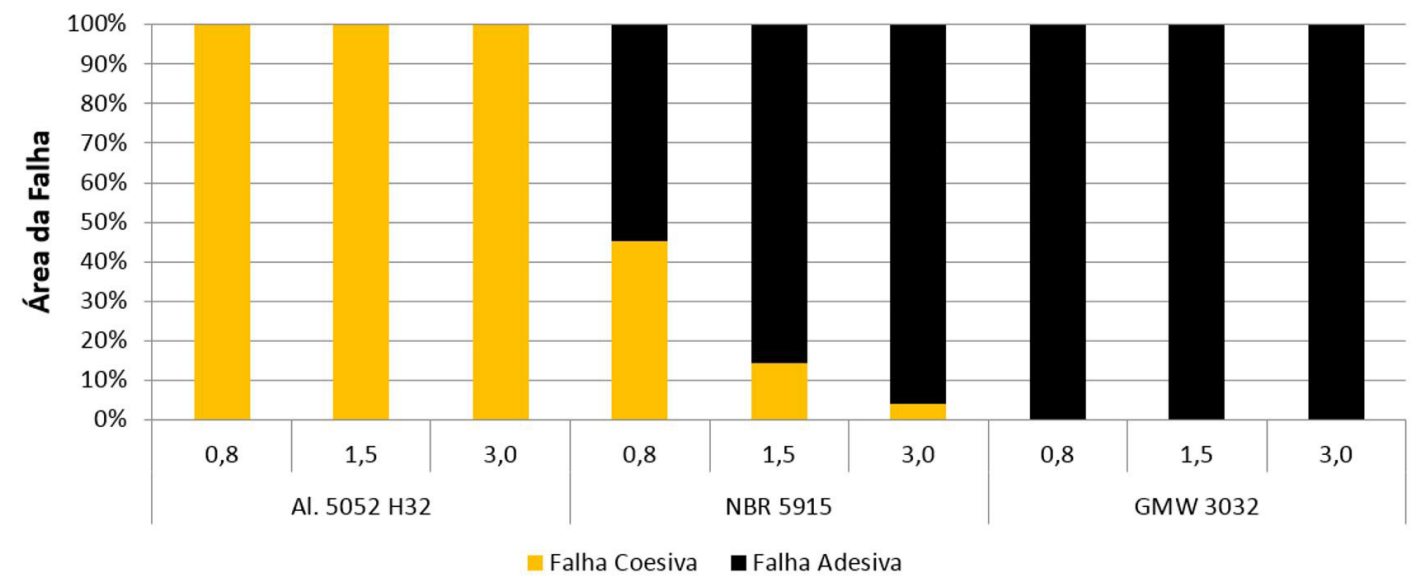

Figura 5. Caracterização dos modos de falhas com diferentes espessuras do adesivo.

propensão a um estado plano de deformações, sendo este responsável pelo favorecimento da fragilidade (brittleness) do material, favorecendo, consequentemente a propagação de trinca. Assim, é possível concluir que a tensão máxima e o máximo deslocamento na junta decrescem significativamente com o aumento da espessura da camada adesiva.

O gráfico da Figura 6 apresenta a média e o desvio padrão referente às medidas de cada uma das condições analisadas com diferentes espessuras do adesivo, referentes à tensão obtida no ensaio de tração cruzada (cross peel).

Através do gráfico da Figura 6, pode-se constatar que com exceção do substrato NBR 5915, o aumento da espessura do adesivo não proporciona uma diminuição linear decrescente da tensão média, fato comprovado pelos resultados obtidos nos ensaios com o alumínio $5052 \mathrm{H} 32$ e o aço galvanizado GMW 3032, que possui tensão média muito próximas quando comparados com as espessuras da camada adesiva de $0,80 \mathrm{~mm}$ e $3,00 \mathrm{~mm}$. O substrato de alumínio $5052 \mathrm{H} 32$ quando submetido a uma espessura da camada adesiva de $0,80 \mathrm{~mm}$ e $3,00 \mathrm{~mm}$ possui um acréscimo de aproximadamente $15 \%$ na tensão média em relação a espessura da camada adesiva com $1,50 \mathrm{~mm}$ de espessura. Já o aço galvanizado GMW 3032 esse acréscimo de tensão da espessura adesiva de $0,80 \mathrm{~mm}$ e $3,00 \mathrm{~mm}$ pode ultrapassar os $50 \%$ em relação a camada adesiva com espessura de I,50mm.

Os motivos pelos quais existe este acréscimo de resistência para espessura do adesivo acima de $1,50 \mathrm{~mm}$ não são bem conhecidos. Crocombe [32] realizou ensaios experimentais, onde mostrou que maiores espessuras proporcionam resistências menores da junta, apesar dos modelos analíticos de Volkersen [33] e Goland Reissner [34] preverem seu aumento. Embora existam teorias que procuram esclarecer o porquê de a diferença de espessura da camada adesiva influenciarem nos valores de resistência, não existe consenso [35].
No aço carbono NBR 5915, pode-se verificar que os valores de tensão dos corpos de prova colados decai conforme aumenta a espessura da camada adesiva. Porém esta diferença não ultrapassa os $10 \%$ quando comparamos as três diferentes espessuras da camada adesiva.

$\mathrm{Na}$ Figura 7, pode-se verificar um exemplo dos modos de falhas ocorridos nos corpos de prova submetidos ao ensaio de tração cruzada (cross peel).

A Figura 8 apresenta o gráfico onde é possível visualizar os diferentes modos de falhas ocorridos nos corpos de prova, caracterizadas como coesiva e adesiva e estão apresentadas na forma de porcentagem de área em que cada falha ocorreu, correspondente a uma área colada de $645,16 \mathrm{~mm}^{2}\left(\right.$ I pol. $\left.{ }^{2}\right)$.

De maneira similar ao encontrado no ensaio de tração cisalhante (lap shear), o substrato em alumínio apresentou em todas as espessuras do adesivo falha $100 \%$ coesiva. Isso indica que a ligação entre $o$ adesivo e o substrato é mais forte que do que a própria resistência interna do adesivo. No entanto, as amostras de aço NBR 59 I 5 possuem regiões com falha mista, ou seja, coesiva e adesiva. Sendo a condição mais favorável em relação aos modos de falha foi com a espessura da camada do adesivo com $1,50 \mathrm{~mm}$, a qual apresentou $38 \%$ de falha coesiva e $62 \%$ de falha adesiva.

Um ponto importante que pode ser observado no gráfico da Figura 8 é em relação ao substrato zincado GMW 3032, que diferentemente dos resultados encontrados no ensaio de tração cisalhante (falhas 100\% adesivas) aqui o mesmo apresentou modo de falha mista (coesiva e adesiva), sendo que a espessura da camada do adesivo influencia diretamente no modo de falha, onde percebe-se um acréscimo da falha adesiva à medida que a espessura aumenta. Segundo Couvrat [36] uma explicação para o surgimento de falha mista é a concentração de tensões não lineares, causada muitas vezes pelo tratamento superficial mal executado, ocasionando primeiramente uma ruptura adesiva, levando a seguir uma ruptura coesiva nas partes da 


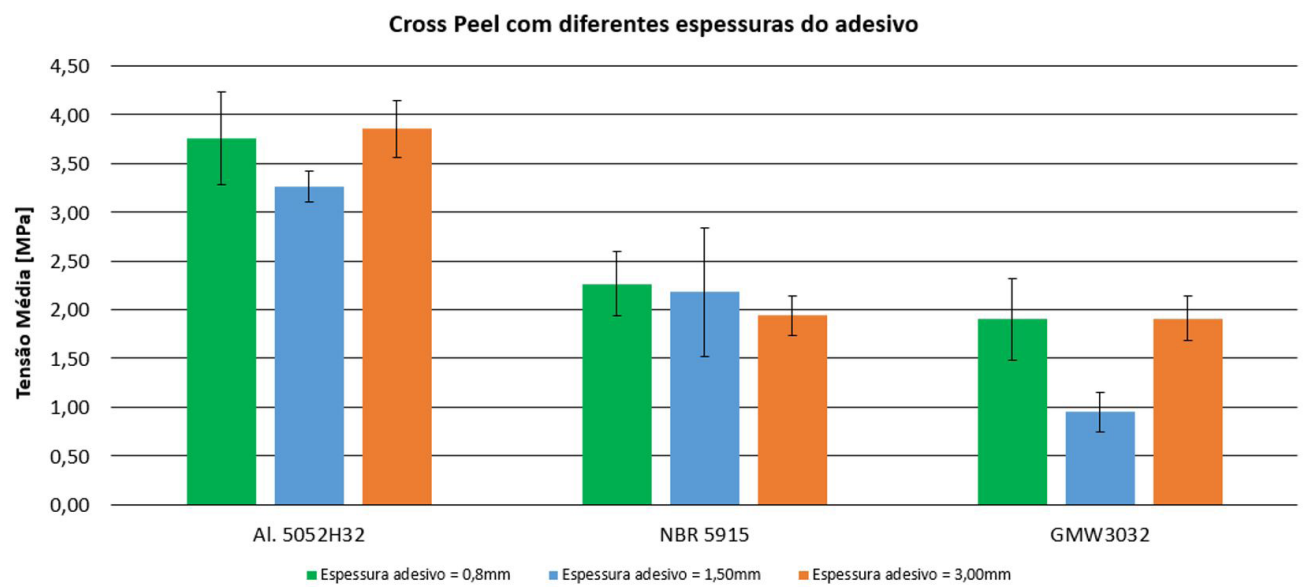

Figura 6. Ensaio de tração cruzada (cross peel).
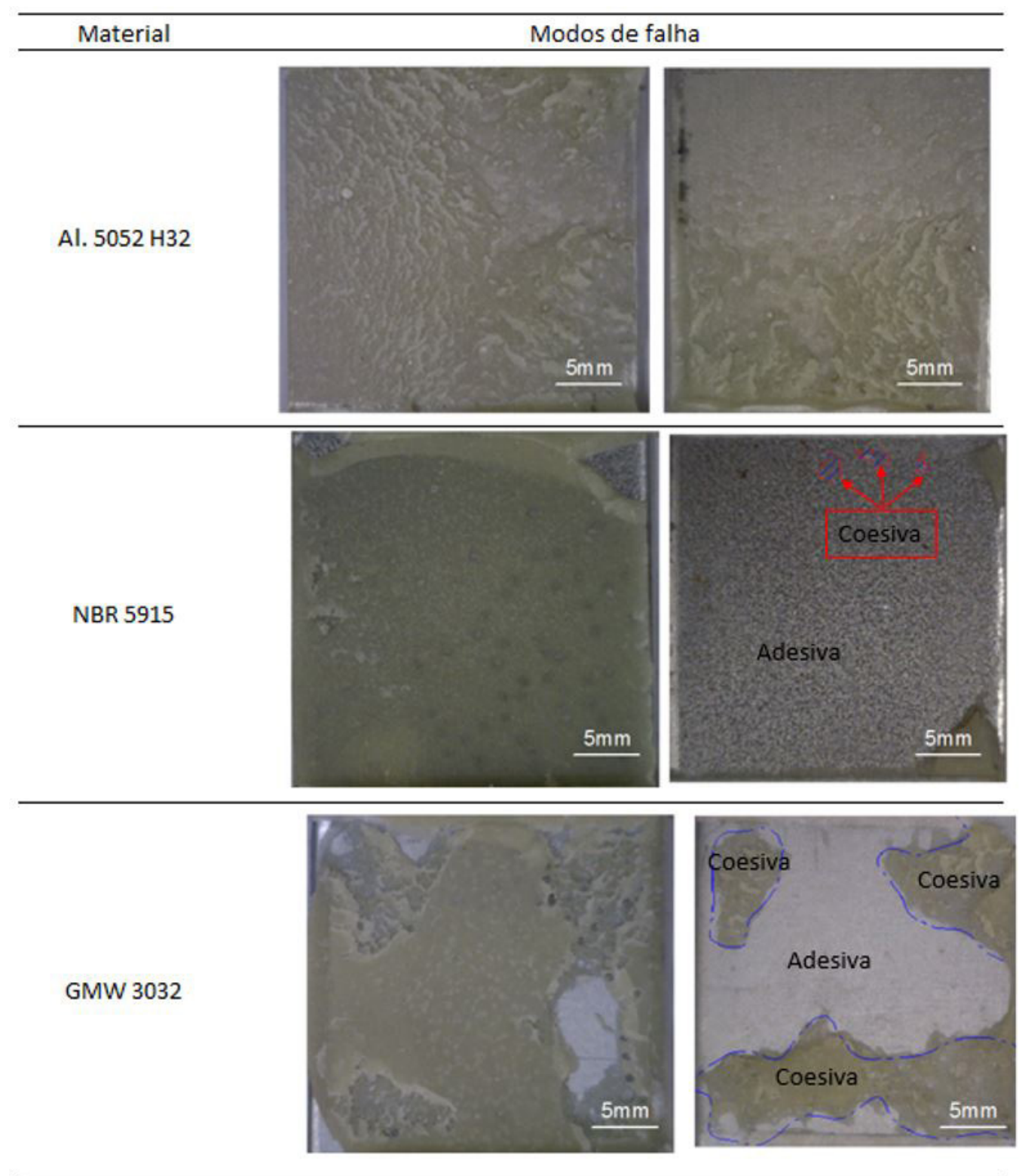

Figura 7. Modos de falhas de tração cruzada (cross peel). 


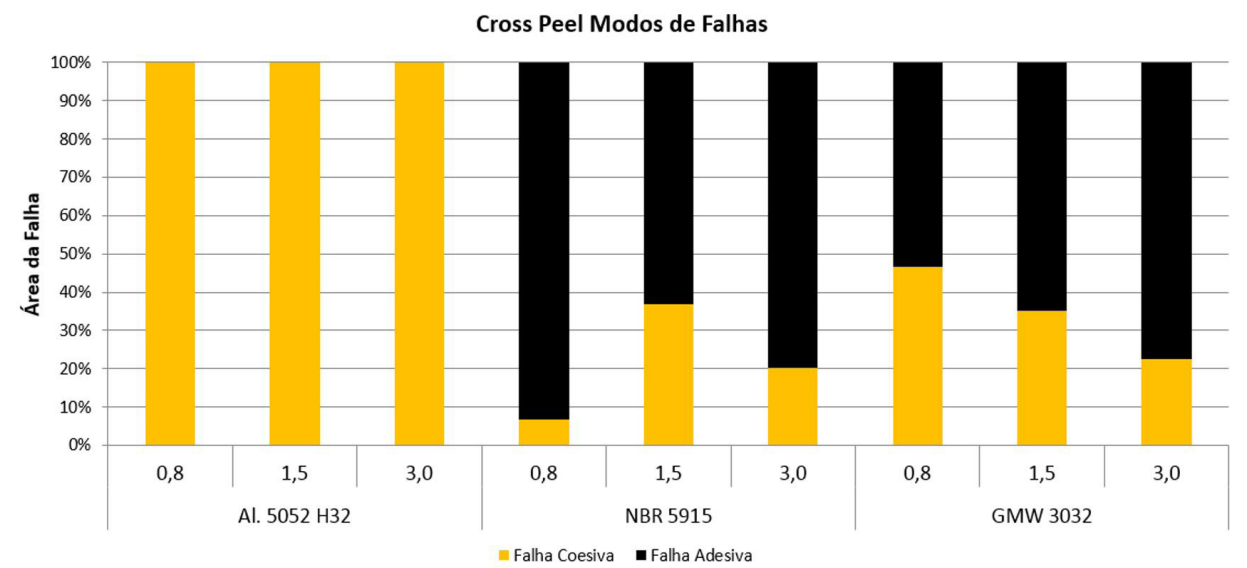

Figura 8. Caracterização dos modos de falhas com diferentes espessuras do adesivo.

superfície que foram bem tratadas. Da Silva e Campilho [37] analisaram as tensões em juntas coladas submetidas a tração pura (cross peel) e constataram que a concentração de tensões pode causar uma distribuição de tensões não lineares, levando ao aparecimento de tensões de delaminação ou clivagem. Desta forma, podemos constatar que para os corpos de prova onde ocorreram falhas mistas, está associado à distribuição de tensões não lineares.

\section{CONCLUSÕES}

Este trabalho teve a finalidade de comparar três diferentes espessuras de adesivo $(0,8,1,5$ e $3,0 \mathrm{~mm}) \mathrm{na}$ adesão de chapas metálicas submetidas a esforços de tração cisalhante (lap shear) e tração cruzada (cross peel). Com base nos resultados obtidos, pode se concluir que:

- Os resultados obtidos no ensaio de lap shear (cisalhamento), mostram que grandes espessuras de adesivo $(e=3,0 \mathrm{~mm})$ suportam menos esforços de ruptura, quando comparados com as mesmas juntas com menor espessura de adesivo $(e=0,8 \mathrm{~mm})$;

- No entanto, o critério de diminuição da resistência da junta adesiva com o aumento da espessura do adesivo não obteve o mesmo comportamento para os resultados obtidos no ensaio de cross peel (tração axial). Como no caso, da junta adesiva com espessura de $1,50 \mathrm{~mm}$, que resistiu menos que a mesma junta com espessura do adesivo de $3,00 \mathrm{~mm}$. Esta conclusão está de acordo com investigações já publicadas sobre o efeito da variação da espessura do adesivo;
- Verificou-se que o tratamento químico de deposição de zinco (galvanização) na superfície do material, compromete a adesão, diminuindo a tensão superficial devido à presença de um elemento intermediário ao material de base, o qual apresenta limitações na capacidade de absorver forças de arrancamento;

- O substrato em alumínio foi o único que apresentou em todas as espessuras do adesivo falha $100 \%$ coesiva, tanto em ensaio de lap shear como cross peel. Isso indica que a ligação entre $o$ adesivo e o substrato é mais forte que do que a própria resistência interna do adesivo;

- De maneira geral, a espessura do adesivo associada a preparação superficial da junta, possui uma importante influência na força máxima que a junta pode suportar.

Por fim, pode-se afirmar que foi possível compreender e avaliar com precisão o comportamento das juntas adesivas, e que existe um grande potencial na utilização de adesivos estruturais na indústria. Porém é importante o conhecimento dos parâmetros que favorecem sua implementação em escala de produção, tornando mais seguro e aplicável este tipo de junta na indústria.

\section{Agradecimentos}

Os autores agradecem às empresas Bruning Tecnometal e LORD Corporation, pelo apoio e colaboração no que diz respeito a este projeto.

\section{REFERÊNCIAS}

I Silva LFM, Neves PJC, Adams RD, Spelt JK. Analytical models of adhesively bonded joints e part I: literature survey. International Journal of Adhesion and Adhesives. 2009;29:319-330.

2 Barnes TA, Pashby IR. Joining techniques for aluminum space frames used in automobiles Part II $Ð$ adhesive bonding and mechanical fasteners. Journal of Materials Processing Technology. 2000;99:72-79. 
3 Adams RD, Comyn J, Wake WC. Structural adhesive joints in engineering. 2nd ed. London: Chapman \& Hall; 1997.

4 Adams RD, Peppiatt NA. Stress analysis of adhesive bonded lap joints. J Strain Anal. 1974;9(3): 185-196.

5 Arenas Reina JM, Narbón PJJ, Alía C. Influence of the surface finish on the shear strength of structural adhesive joints and application criteria in manufacturing processes. The Journal of Adhesion. 2009;85(6):324-340.

6 Xin Y, Li Y, Yong X, Qing Z. Effect of base steels on mechanical behavior of adhesive joints with dissimilar steel substrates. International Journal of Adhesion and Adhesives. 2014;51:42-53.

7 De'Nève B, Delamar M, Nguyen TT, Shanahan MER. Failure mode and ageing of steel/epoxy joints. Applied Surface Science. 1998; 134:202-2I2.

8 Challita G, Othman R, Casari P, Khalil K. Experimental investigation of the shear dynamic behavior of double-lap adhesively bonded joints on a wide range of strain rates. International Journal of Adhesion and Adhesives. 2011;31:146-153.

9 Seo DW, Lim JK. Tensile bending and shear strength distributions of adhesive bonded butt joint specimens. Composites Science and Technology. 2005;65: I 42 I- 1427.

10 Peroni L, Avalle M, Belingardi G. Comparison of the energy absorption capability of crash boxes assembled by spotweld and continuous joining techniques. International Journal of Impact Engineering. 2009;36:498-5I I.

I I Seong MS, Kim TH, Nguyen KH, Kweon JH, Choi JH. A parametric study on the failure of bonded single-lap joints of carbon composite and aluminum. Composite Structures. 2008;86:I35-I45.

12 Silva LFM, Magalhães AG, Moura MFSF. Juntas adesivas estruturais. Porto: Publindústria; 2007.

13 Schijve J, Van Lipzig HTM, Van Gestel GFJA, Hoeymakers AHW. Fatigue properties of adhesive-bonded laminated sheet material of aluminum alloys. Engineering Fracture Mechanics. 1979;12:56I-579.

14 Her SC. Stress analysis of adhesively-bonded lap joint. Composite Structures. 1999;47:673-678.

15 Li G, Lee-Sullivan P, Thring RW. Nonlinear finite element analysis of stress and strain distributions across the adhesive thickness in composite single-lap joints. Composite Structures. 1999;46:395-403.

16 Pandey PC, Shankaragouda H, Singh AK. Nonlinear analysis of adhesively bonded lap joints considering viscoplasticity in adhesives. Composite Structures. 1999;70:387-4I3.

17 Vaidya UK. Experimental-numerical studies of transverse impact response of adhesively bonded lap joints in composite structures. International Journal of Adhesion and Adhesives. 2006;26:184- 198.

18 American Society for Testing and Materials - ASTM. ASTM D 1002. Standard Teste Method for Apparent Shear Strenght of Single-Lap Joint Adhesively Bonded Metal Specimens by Tension Loading (Metal-to-Metal). West Conshohocken: ASTM; 1994.

19 SAE International. SAE J1553. Cross Peel Test For Automotive - Type Adhesives for Fiber Reinforced Plastic [FRP] Bonding. SAE The Engineering Resource for Advancing Mobility, 400 Commonwealth Drive. Warrendale: SAE International; 1986.

20 Associação Brasileira de Normas Técnicas - ABNT. ABNT NBR 6834. Alumínio e suas ligas - Classificação da Composição Química. Rio de Janeiro: ABNT; 2006.

2I Associação Brasileira de Normas Técnicas - ABNT. ABNT NBR 7823. Alumínio e suas ligas - Chapas - Propriedades Mecânicas. Rio de Janeiro: ABNT; 2007.

22 General Motors Worldwide. GMW3032. High strength sheet steel, 180 MPa through 700 Mpa yield strenghts. USA: General Motors Worldwide. Worldwide Engineering Standards; 2013.

23 Associação Brasileira de Normas Técnicas - ABNT. ABNT NBR 59I5. Bobinas e chapas finas a frio de aço carbono para estampagem - Especificação. Rio de Janeiro: ABNT; 2008.

24 LORD Corporation. Dados técnicos. Adesivos acrílicos LORD 403, 406 e 410 com acelerador 19 ou $19 \mathrm{~GB}$ da LORD. Cary: LORD Corporation; 20 I2. NC 275I I-7923.

25 Mahdi S, Kinloch AJ, Matthews CMA. The static mechanical performance of repaired composite sandwich beams: part I; experimental characterization. The Journal of Sandwich Structures \& Materials. 2003;5: 179-202.

26 Naito K, Onta M, Kogo Y. The effect of adhesive thickness on tensile and shear strength of polyimide adhesive. International Journal of Adhesion and Adhesives. 2012;36:77-85.

27 Adams RD, Peppiatt N. J Strain Anal. 1974;9:185.

28 Gleich DM, Van Tooren MJL, Beukers A. Journal of Adhesion Science and Technology. 200 I;I5:109I. 
Almeida et al.

29 Fruet TF. Análise para substituição da solda ponto de uma carroceria de chapas dinas de aço carbono e aço galvanizado por adesivo estrutural [trabalho de conclusão]. Caxias do Sul: Universidade de Caxias do Sul; 20I I.

30 Bikerman, J.J. Particle adhesion theory and experiment. Advances in Colloid and Interface Science. 1967; I: I I I-239.

3I Abdelaziz AT, Rachid B, Said A, Sebastien G, Hychem B. Bonded joints with composite adherends. Part I - Effect of specimen configuration, adhesive thickness, spew fillet and adherend stiffness on fracture. International Journal of Adhesion and Adhesives. 2006;26(4):226-236.

32 Crocombe AD. Global yielding as a failure criteria for bonded joints. International Journal of Adhesion \& Adhesives. 1989:|45-I53.

33 Volkersen O. Die nietkraftoerteilung in zubeanspruchten nietverbindungen mit konstanten loschonquerschnitten. Luftfahrtforschung. 1938;15:41-47.

34 Goland M, Reissner E. The stresses in cemented joints. Journal of Applied Mechanics. 1944:AI7-A27.

35 Silva LFM, Campilho RDSG. Design of adhesively-bonded composite joints. In: Vassilopoulos AP. Fatigue and fracture of adhesively-bonded composite joints: behaviour, simulation and modelling. Kidlington: Woodhead Publishing; 2015.

36 Couvrat P. Le collage structural moderne: théorie et pratique. Paris: Tec \& DocLavoisier; 1992.

37 Silva LFM, Campilho Raul DSG. Advances in numerical modeling of adhesive joints. Berlin: Springer Berlin Heidelberg; 2012.

Recebido em: 22 Fev. 2017

Aceite em: 14 Nov. 2018 